\title{
Prokineticin 2 Modulates the Excitability of Subfornical Organ Neurons
}

\author{
G. Trevor Cottrell, ${ }^{1}$ Qun-Yong Zhou, ${ }^{2}$ and Alastair V. Ferguson ${ }^{1}$ \\ ${ }^{1}$ Department of Physiology, Queen's University, Kingston, Ontario, Canada K7L 3N6, and 2Department of Pharmacology, University of California, Irvine, \\ California 92697
}

The recent discovery of prokineticin 2 (PK2) expression in the suprachiasmatic nucleus and its receptors in critical autonomic control centers of the brain, including the subfornical organ (SFO), suggests the intriguing possibility that PK2 regulates the excitability of SFO neurons and thus influences autonomic function. Using current-clamp techniques to record from dissociated SFO neurons, we examined the effects of PK2 on the excitability of these cells. PK2 (20 nM) induced depolarizations in 40\% of SFO neurons ( $n=45$; mean, $7.5 \pm 1.7$ $\mathrm{mV}$ ), an effect that was reversible, PK2-specific, and concentration dependent. The depolarization was accompanied by an increase in action potential frequency from $0.4 \pm 0.1$ to $1.4 \pm 0.5 \mathrm{~Hz}$ in responding cells $(n=10)$. This excitatory effect appears to be, in part, attributable to a PK2-induced decrease in the delayed rectifier potassium current $\left(I_{\mathrm{K}}\right)$. In $10 \mathrm{SFO}$ neurons recorded using perforated patch voltage-clamp techniques, six demonstrated a reversible decrease in $I_{K}$ (mean decrease, $26.7 \pm 6.4 \%$ ) in response to 20 nм PK2, whereas artificial CSF alone was without an effect on these currents. These data are the first to show excitatory effects of PK2 on neurons and, in addition, demonstrate that this peptide modulates voltage-activated $\mathrm{K}^{+}$channels. The activation of SFO neurons by PK2 illustrates a mechanism through which this peptide may exert circadian control of autonomic functions.

Key words: autonomic; circadian; circumventricular; neuropeptide; patch clamp; potassium [K]

\section{Introduction}

Prokineticin 2 (PK2) is a novel 81 amino acid peptide with a complex folding structure including five disulphide linkages. It is a mammalian homolog to the mamba snake venom protein mamba intestinal toxin 1 and the frog protein $\mathrm{Bv} 8$, and has been demonstrated to be a paracrine mediator of gastrointestinal smooth muscle contractility (Li et al., 2001). Prokineticin 1 (PK1), a homolog of PK2, is also identical to endocrine glandderived vascular endothelial growth factor, a potent angiogenic mitogen in secretory organs (LeCouter et al., 2001; Masuda et al., 2002).

PK2 is expressed throughout the mammalian organism; however, in the brain, its expression is specifically concentrated in the suprachiasmatic nucleus (SCN) (Cheng et al., 2002). PK2 is rhythmically expressed in the SCN, transcriptionally regulated by clock control genes, and has the capacity to strongly suppress nocturnal locomotor activity. PK2 receptors are located in the SCN as well as numerous autonomic control centers in the brain such as the paraventricular nucleus (PVN) and the subfornical organ (SFO) (Cheng et al., 2002). PK2 is one of the first neuropeptides that appears to directly convey circadian rhythm sig-

Received July 23, 2003; revised Jan. 19, 2004; accepted Jan. 19, 2004

This work was supported by the Heart and Stroke Foundation of Canada (G.T.C.) and the Canadian Institutes for Health Research.

Correspondence should be addressed to Dr. Alastair V. Ferguson, Queen's University, Department of Physiology, Botterell Hall, Fourth Floor, Kingston, Ontario, Canada K7L 3N6. E-mail: avf@post.queensu.ca. DOI:10.1523/JNEUROSCI.5187-03.2004

Copyright $\odot 2004$ Society for Neuroscience $\quad$ 0270-6474/04/242375-05\$15.00/0 naling to other CNS structures and, therefore, it may be a critical output molecule regulating biological rhythms.

The SFO is a circumventricular organ structure that has essential actions in the integrative regulation of cardiovascular, immune, feeding, and reproductive function (McKinley et al., 1998; Cottrell and Ferguson, 2004). It is ideally situated in the forebrain to be exposed to both blood-borne signals and those circulating within the CNS. The role of the SFO in the regulation of cardiovascular function has been well characterized. It is sensitive to stimulation by angiotensin II (ANG) and vasopressin and is intrinsically osmosensitive. The SFO influences cardiovascular function through projections to the hypothalamus and other autonomic control centers (Lind et al., 1982; Ferguson and Renaud, 1986).

The integrative action of the SFO makes it an ideal regulatory target for signaling molecules designed to influence autonomic function. As a result, it could play a significant role in PK2 signaling from either the periphery or the CNS. The purpose of these experiments was to determine whether the exposure of SFO neurons to PK2 resulted in increased neuronal excitability. We demonstrated that PK2 is capable of depolarizing SFO neurons and increasing their firing frequency. This effect was at least partially mediated by an inhibition of the delayed rectifier $\mathrm{K}^{+}$current but not the transient outward $\mathrm{K}^{+}$current.

\section{Materials and Methods}

Chemicals. Unless otherwise indicated, all chemical reagents were from Sigma (St. Louis, MO). PK2 was produced using recombinant techniques described previously (Li et al., 2001) and was used at a standard concentration of $20 \mathrm{~nm}$ for all experiments unless otherwise indicated. This 
concentration corresponds to the top range of the dose-response for PK2 effects in gastrointestinal smooth muscle and is similar to that used to induce calcium transients in neurons (Li et al., 2001; Negri et al., 2002). The PK2 antagonist A1MPK1 was produced using recombinant techniques described previously (Bullock et al., 2004). A1MPK1 is an effective inhibitor of PK2 action when at a 40-fold greater concentration then PK2. Nystatin stock solution ( $100 \mathrm{gm} / \mathrm{l}$ in dimethylsulfoxide) was made fresh each day and stored at $4^{\circ} \mathrm{C}$ until use in experiments when it was then suspended in pipette solution (see Electrophysiology) at a final concentration ranging from 100 to $250 \mu \mathrm{g} / \mathrm{ml}$. DMSO content in the internal solution was never $>0.03 \%$. Tetrodotoxin (TTX; Alomone Labs, Jerusalem, Israel) was stored as a stock solution in $1 \mathrm{~mm}$ aliquots and made fresh daily by suspension in artificial CSF (aCSF) to a final concentration of $100 \mathrm{~nm}$.

Subfornical organ neuron dissociation. Experiments with animals were approved by the Queen's University Animal Care Committee. The SFO dissociation procedure was performed as described previously (Ferguson et al., 1997). Male Sprague Dawley rats (125-175 gm) were decapitated, and the brains were removed and immediately immersed in ice-cold $\mathrm{Ca}^{2+}$ - and $\mathrm{Mg}^{2+}$-free HBSS (Invitrogen, Grand Island, NY) supplemented with $0.09 \mathrm{M}$ sucrose. A tissue block containing the hippocampal commissure and SFO was dissected free from the brain, and the SFO was separated from the surrounding tissue. The isolated SFO was immersed in $1 \mathrm{mg} / \mathrm{ml}$ Trypsin and incubated at $37^{\circ} \mathrm{C}$ in $5 \% \mathrm{CO}_{2}, 95 \% \mathrm{O}_{2}$ for $30 \mathrm{~min}$. Cells were then suspended in ice-cold $\mathrm{Ca}^{2+}$ containing HBSS (Invitrogen) supplemented with $4 \mu \mathrm{g} / \mathrm{ml} \mathrm{BSA}$, triturated through a 20 gauge needle using a $1 \mathrm{ml}$ syringe, and centrifuged at $900 \times g$ for $5 \mathrm{~min}$. The supernatant was then removed, cells were resuspended, and a second 5 min centrifugation was performed. Cells were resuspended, plated in culture dishes (Corning, Corning, NY) with a Neuorobasal A Medium (Invitrogen), and incubated for a minimum of $24 \mathrm{hr}$ before electrophysiology experiments.

Electrophysiology. Whole-cell patch-clamp recordings were performed as reported previously (Washburn et al., 1999; Desson and Ferguson, 2003). Electrodes were fabricated from $1.2 \mathrm{~mm}$ filament glass (A-M Systems, Carlsborg, WA) on a Sutter Instruments (Novato, CA) puller and were filled with the following (in $\mathrm{mM}$ ): 130 potassium gluconate, 10 HEPES, 10 EGTA, $1 \mathrm{MgCl}_{2}, 4 \mathrm{Na}_{2} \mathrm{ATP}$, and 0.1 GTP (290-300 mOsm, $\mathrm{pH}$ 7.2). For perforated patch experiments, $10 \mathrm{~mm} \mathrm{KCl}$ was added to the internal solution. The cells were bathed in an external solution (aCSF) containing (in mM): $140 \mathrm{NaCl}, 5 \mathrm{KCl}, 1 \mathrm{MgCl}, 10$ HEPES, 10 glucose, and $2 \mathrm{CaCl}_{2}$ (290-300 mOsm, pH 7.2).

Whole-cell recordings were obtained from SFO cells identified as neurons on the basis of the presence of voltage-gated $\mathrm{Na}^{+}$currents under voltage-clamp conditions, and at least $80 \mathrm{mV}$ action potentials induced at a holding potential below $-55 \mathrm{mV}$ in current-clamp recordings. Signals were amplified using an Axopatch 200B amplifier (Axon Instruments, Foster City, CA) filtered with an 8-pole Bessel filter at $1 \mathrm{kHz}$ and digitized using a Cambridge Electronics Design micro1401 interface (Cambridge Electronics Design, Cambridge, UK) at $8 \mathrm{kHz}$. Data were collected using Signal (voltage clamp) or Spike 2 (current clamp) software packages (Cambridge Electronics Design).

To minimize current rundown during voltage-clamp experiments, perforated patch recordings were performed with electrodes back-filled with Nystatin (see Reagents). After making cell contact with the electrode, light suction was applied to achieve a high resistance seal $(\sim 2-8$ $\mathrm{G} \Omega$ ). Seal quality and cell access were monitored using $-10 \mathrm{mV}$ pulses from a holding potential of $-65 \mathrm{mV}$. Once series resistance was below 30 $\mathrm{M} \Omega$, outward currents were monitored over time until cell access plateaued ( $\sim 15 \mathrm{~min}$ ). Sudden changes in series resistance were monitored between recordings, and electrode suction was applied at the end of experiments to ensure that the perforated patch configuration was patent throughout the experiments. All voltage-clamp experiments were performed in the presence of $100 \mathrm{~nm}$ TTX.

Statistical analysis. In current-clamp recordings, cells were classified as responders if they showed sustained changes $(>60 \mathrm{sec})$ in membrane potential (Em) of $>5 \mathrm{mV}$ in the $1-3 \mathrm{~min}$ period after the initiation of PK2 perfusion. This measure was made relative to the $60 \mathrm{sec}$ period immediately before treatment. The number of responding cells in the PK2 treat-
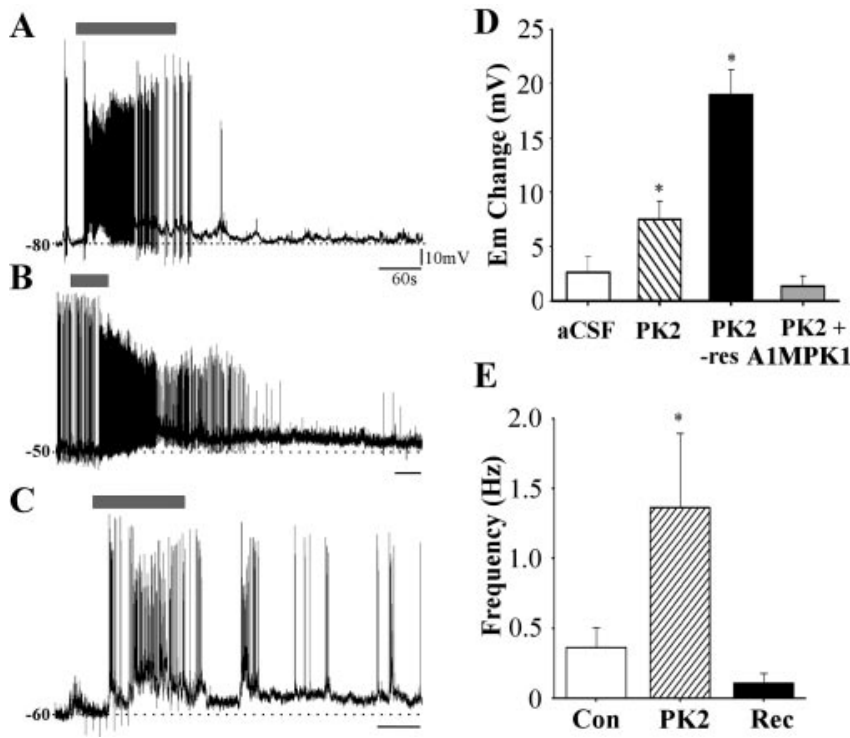

Figure 1. PK2 depolarizes SF0 neurons and increases action potential firing frequency. $A-C$, Current-clamp recordings demonstrating the effect of $20 \mathrm{~nm}$ PK2 on whole-cell membrane potential. The time period of PK2 application is depicted by the gray bars. All horizontal scale bars represent $60 \mathrm{sec}$. D, Mean change in membrane potential measured relative to baseline during treatment with either aCSF (white bar; $n=14$ ), $20 \mathrm{~nm} \mathrm{PK2} \mathrm{(hatched} \mathrm{bar;} n=45$ ), or 1 пм PK2 in combination with $40 \mathrm{~nm}$ A1MPK1 (gray bar; $n=9$ ). The black bar represents the mean depolarization of those SFO neurons classified as responders (PK2-res; $n=18$ ). Asterisks indicate a significant difference compared with aCSF treatment $(p<0.05)$. E, PK2 increased the firing frequency of 7 of 10 cells that demonstrated a reversible PK2 effect on membrane potential. Con (white bar), Firing frequency recorded in the last minute of baseline recording; PK2 (hatched bar), firing frequency recorded 2-3 min after 20 nм PK2 treatment; Rec, firing frequency recorded in the last minute of the washout period. Asterisks indicate a significant effect compared with the baseline measure.

ment group was compared with control aCSF treatment using a $\chi^{2}$ test. Changes in membrane potential and action potential frequency were compared between control and treatment groups using a Student's $t$ test. The concentration-response curve was constructed from a sigmoidal function of the following form:

$$
Y=Y \min +\frac{(Y \max -Y \min )}{1+10^{[\log \mathrm{EC} 50-\log (\mathrm{PK} 2)] k}}
$$

In voltage clamp, cells were distinguished as responders on the basis of a $10 \%$ decrease in current followed by at least a partial recovery toward baseline after an aCSF wash. Responders were compared between control and treatment groups using a $\chi^{2}$ test to assess a treatment effect. Peak current change was compared between control and treatment groups using a Student's $t$ test. Current values in all $I-V$ plots were normalized to the peak current measured during baseline recording. Normalized current change at each voltage step was compared between baseline and treatment conditions using a paired $t$ test. All values are plotted as means \pm SEM with $p$ values set at 0.05 .

\section{Results}

\section{PK2 depolarizes subfornical organ neurons}

The effects of $20 \mathrm{~nm}$ PK2 treatment on membrane potential were assessed during continuous current-clamp recording. In 45 cells recorded, there was a depolarizing effect of PK2 on 18 cells (40\%) with this effect being reversible in $85 \%$ of these responding cells (Fig. $1 A-C$ ). In 14 cells treated with a control aCSF wash, only three had a "response" on the basis of the imposed criteria for depolarization (see Materials and Methods); however, none of these cells had any recovery toward baseline after a second aCSF wash. $\chi^{2}$ comparison of the control and treatment groups shows that the number of responders in the PK2 group was significantly 


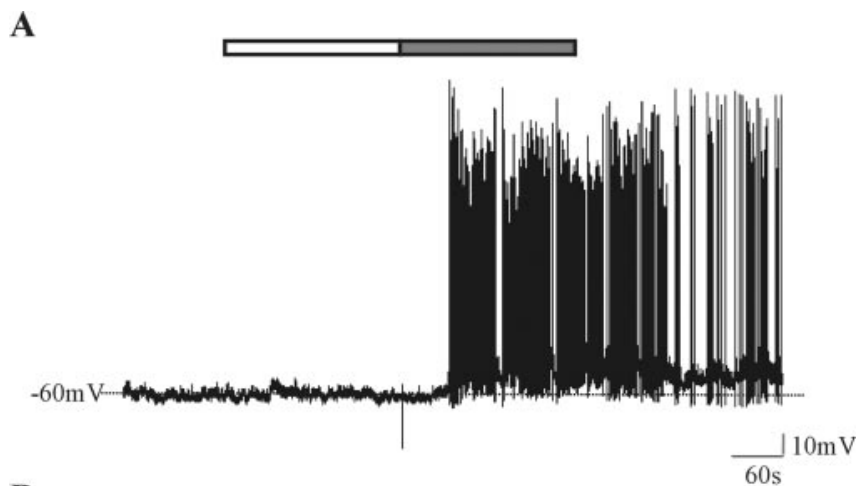

B

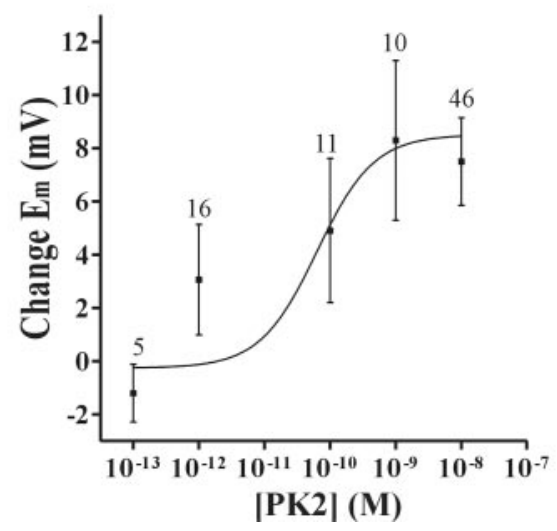

Figure 2. PK2 depolarizes SF0 neurons in a concentration-dependent manner. A, Currentclamp recording during $10^{-13} \mathrm{MPK} 2$ treatment (white bar) followed by $10^{-9} \mathrm{M}$ PK2 treatment (gray bar). $B$, Concentration-response curve for PK2 effects on membrane potential. The $\mathrm{EC}_{50}$ was calculated at 63 pm. Numbers above the data points indicate sample size at each dose.

greater than that expected under control conditions, thus implying that there is a distinct population of SFO cells capable of responding to $\mathrm{PK} 2$.

The mean depolarization of all SFO cells $(n=45)$ in response to $20 \mathrm{~nm}$ PK2 was $7.5 \pm 1.7 \mathrm{mV}$, a value significantly greater than that of the control group $(n=14 ; 2.8 \pm 1.1 \mathrm{mV})$ (Fig. $1 D)$. The mean depolarization of cells identified as responders was $19.0 \pm$ $2.2 \mathrm{mV}(n=18)$ (Fig. 1D). To ensure that the PK2 effects observed were the direct result of PK2 action and not that of a contaminant, the PK2 receptor antagonist A1MPK1 was used. Perfusion of SFO neurons with $40 \mathrm{nM}$ A1MPK1 5 min before perfusion with $1 \mathrm{nM}$ PK2 prevented any depolarization (Fig. $1 D$; see also Fig. 2 for $1 \mathrm{~nm}$ PK2 effect). In the 10 cells demonstrating a reversible $\mathrm{PK} 2$ effect, there was an increase in the action potential firing frequency in seven of the cells with the mean firing frequency being almost fourfold greater after PK2 treatment when compared with baseline values (control, $0.4 \pm 0.1$ vs PK2, $1.4 \pm 0.5 \mathrm{~Hz} ; n=10 ; p<0.05$ ) (Fig. $1 E$ ). Changes in membrane potential were concentration dependent, with peak depolarization occurring at $1 \mathrm{~nm}$ PK2, no response being observed at $1 \mathrm{fM}$, and an $\mathrm{EC}_{50}$ of 63 pM (Fig. 2).

PK2 inhibits delayed rectifier $\mathrm{K}^{+}$current $\left(I_{\mathrm{K}}\right)$

Previous research has demonstrated a pattern of hyperexcitability of SFO neurons induced by external ligands, with this effect being mediated in part by the inhibition of outward $\mathrm{K}^{+}$currents (Ferguson and Li, 1996; Washburn et al., 1999). Based on this, and a decreased afterhyperpolarization amplitude and duration following action potentials in some of the responding cells, we ex-
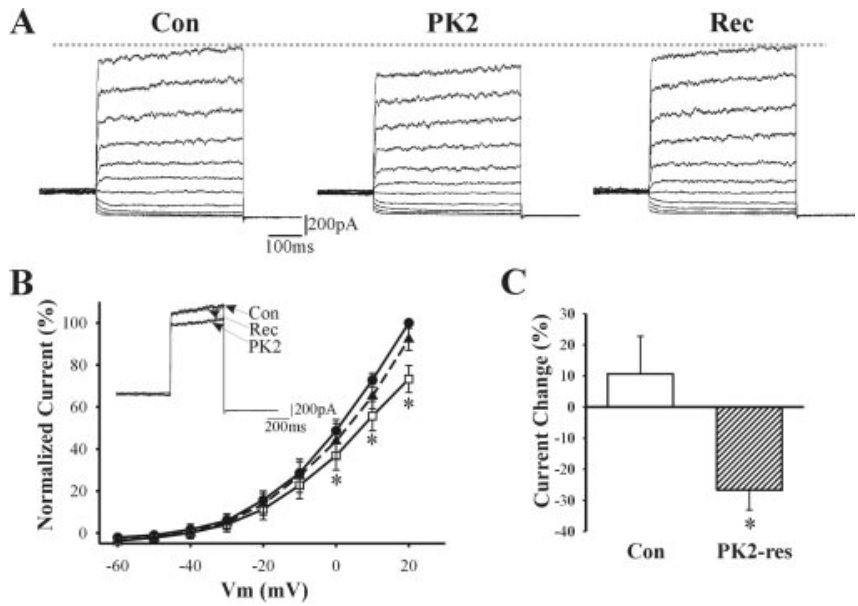

C

Figure 3. PK2 inhibits $I_{K}$ current. $A$, Voltage-clamp recordings of $I_{K}$ currents produced by taking $10 \mathrm{mV}$ steps between -80 and $20 \mathrm{mV}$ from a holding potential of $-40 \mathrm{mV}$. Current traces represent baseline (Con), $20 \mathrm{~nm}$ PK2 treatment (PK2), and PK2 washout (Rec) conditions in the same neuron. $B, I-V$ plot of normalized steady-state current, averaged across experiments $(n=6)$ and plotted at each voltage step for the control period (filled circles) during $20 \mathrm{~nm}$ PK2 treatment (open squares) and after PK2 washout (filled triangles). The PK2 treatment effect was first observed at the $0 \mathrm{mV}$ step. Asterisks indicate a significant difference from control ( $p<$ 0.05 ). Inset, Overlaid current traces from pulses to $20 \mathrm{mV}$ during control, PK2, and recovery periods. C, Mean change in current measured at the $20 \mathrm{mV}$ pulse for an aCSF wash (white bars; $n=7$ ) and 20 nм PK2 treatment (hatched bars; $n=6$ ). The mean PK2 current change was derived from neurons defined as responders. Asterisks indicate a significant difference from the aCSF treatment $(p<0.05)$.

amined the effects of PK2 on delayed rectifier $\left(I_{\mathrm{K}}\right)$ and transient outward $\left(I_{\mathrm{A}}\right) \mathrm{K}^{+}$currents.

Voltage-clamp recordings using perforated patch techniques were used to isolate $I_{\mathrm{K}}$ from the $I_{\mathrm{A}}$ by administering $10 \mathrm{mV}$ voltage steps between -80 and $20 \mathrm{mV}$ from a $500 \mathrm{msec}$ prepulse step of $-40 \mathrm{mV}$, a protocol which inactivates $I_{\mathrm{A}}$ and thus permits direct assessment of $I_{\mathrm{K}}$ (Washburn et al., 1999). In 10 cells recorded, six demonstrated a decrease in $I_{\mathrm{K}}$ current, which was reversible after washout with aCSF (Fig. $3 A$ ). The number of responding cells in the $\mathrm{PK} 2$ treatment group was significantly greater than a control aCSF wash (Con), where no cells ( 0 of 7 ) demonstrated a response on the basis of the criteria established (see Materials and Methods).

Comparison of $I-V$ relationships shows a significant decrease in $I_{\mathrm{K}}$ at the $0 \mathrm{mV}$ step, with the current difference increasing progressively up to the $20 \mathrm{mV}$ step after $20 \mathrm{nM}$ PK2 treatment (Fig. 3B). The mean change in current at the $20 \mathrm{mV}$ step was significantly greater in the PK2-treated cells labeled responders ( $26 \pm 6 \%$ decrease) when compared with control aCSF perfusion for the same time period ( $10 \pm 12 \%$ increase; $p<0.05$ ) (Fig. $3 C$ ). There were no differences in mean $I_{\mathrm{K}}$ change between the control-treated cells and the PK2-treated cells identified as nonresponders (19 $\pm 13 \%$ increase; data not shown).

\section{PK2 has no effect on transient outward $\mathrm{K}^{+}$current $\left(I_{\mathrm{A}}\right)$}

$I_{\mathrm{A}}$ was pharmacologically isolated from sustained outward currents in SFO neurons using aCSF containing $10 \mathrm{~mm}$ TEA. Voltage-clamp protocols incorporated $500 \mathrm{msec}, 10 \mathrm{mV}$ steps between -100 and $20 \mathrm{mV}$ from a holding potential of $-100 \mathrm{mV}$. These steps resulted in rapidly activating, rapidly inactivating currents typical of $I_{\mathrm{A}}$ (Fig. 4A). PK2 had no effect on this isolated $I_{\mathrm{A}}$ current, with no significant change in normalized current being recorded at any voltage step after treatment (Fig. $4 \mathrm{~B}$ ). This effect was identical to a control aCSF perfusion for a similar time 
A
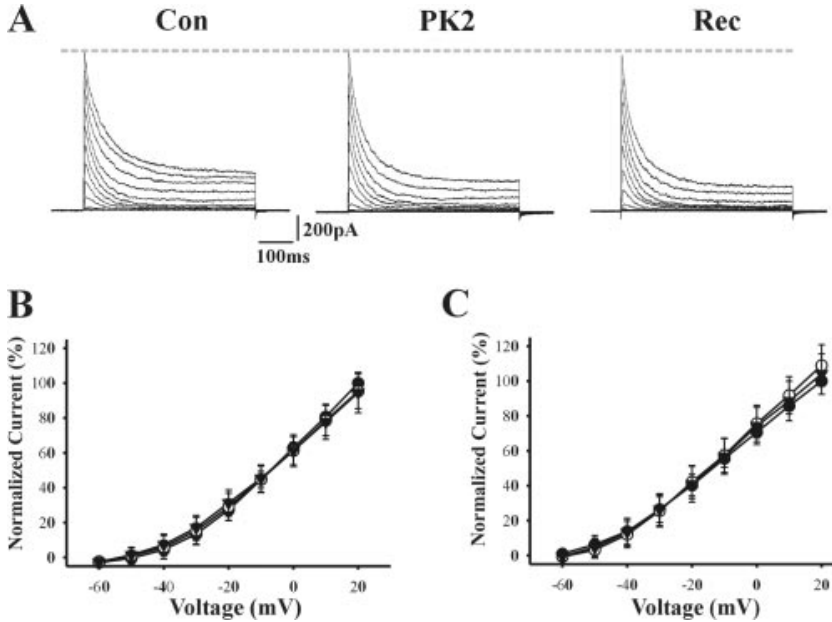

C

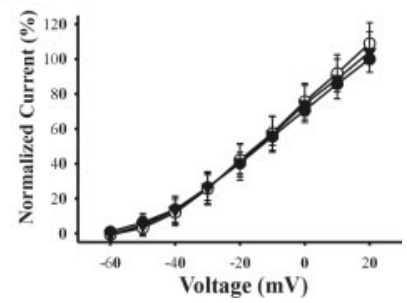

Figure 4. PK2 has no effect on $I_{A}$ current. $A$, Voltage-clamp recordings of $I_{A}$ currents produced by taking $10 \mathrm{mV}$ steps between -100 and $20 \mathrm{mV}$ from a holding potential of $-100 \mathrm{mV}$ in the presence of $10 \mathrm{~mm}$ TEA. Current traces represent baseline (Con), $20 \mathrm{~nm}$ PK2 treatment (PK2), and PK2 washout (Rec) conditions in the same neuron. B, I-V plot of normalized peak current, averaged across experiments $(n=7)$ and plotted at each voltage step for the control period (filled circles) during $20 \mathrm{~nm}$ PK2 treatment (open circles) and after PK2 washout (filled triangles). $C, I-V$ plot of normalized peak current, averaged across experiments $(n=5)$ and plotted at each voltage step for the control period (filled circles) and during consecutive aCSF treatments (open circles, closed triangles).

period (Fig. 4C), with the mean change in current at the $20 \mathrm{mV}$ step being $9.0 \pm 12 \%(n=5)$, as compared with $4.7 \pm 10 \%$ in the PK2 treatment group $(n=7)$.

\section{Discussion}

These data are the first to demonstrate an effect of PK2 on the excitability of CNS neurons. PK2 depolarized $40 \%$ of the SFO neurons tested and increased the action potential firing frequency in the majority of these cells. It is also the first time PK2 has been demonstrated to have an effect on voltage-gated $\mathrm{K}^{+}$ $\left(K_{\mathrm{v}}\right)$ channels. This observation could account for the increase in action potential firing frequency in PK2-treated neurons, although not necessarily for the membrane depolarizations observed at hyperpolarizing holding potentials, thus suggesting that PK2 influences other membrane properties. These findings demonstrate a neuromodulatory role for PK2 and identify the SFO as a potential site for circadian regulation of autonomic function.

PK2 is expressed both centrally and in the periphery. In the periphery, its expression is focused in gastrointestinal tissues at levels significantly lower than PK1. In this region, PK2 has a paracrine effect on the stimulation of gastrointestinal smooth muscle contraction (Li et al., 2001). Centrally, PK2 expression is focused in the SCN; however, the median preoptic area, nucleus accumbens, and islands of Calleja also contain PK2 mRNA signal (Cheng et al., 2002). Two studies have examined the effects of the PK2 orthologue, Bv8, on neuronal function and determined that it increases nociceptive sensitization of the tail and paw in rats, increases intracellular calcium in small diameter dorsal root ganglion neurons in culture (Negri et al., 2002), and supports neuronal survival (Melchiorri et al., 2001). Only the original work by Cheng et al. (2002) examined PK2 action in the brain.

The source of PK2 that modulates the SFO is not clear. To date, there is no evidence for direct efferent $\mathrm{SCN}$ projections to the SFO, although it is possible that the detection sensitivity of methodologies used thus far is not sufficient to identify such interactions. Because the SFO has the unique characteristic of being a sensory circumventricular organ, it could receive PK2 signaling through a number of different pathways such as from the periphery via the blood, from another brain region such as the median preoptic area, from the SCN via diffusion through the CSF, or through direct SCN efferents that have not yet been identified.

Although the physiological implications of PK2 effects on SFO neurons are not clear, the SFO has well established roles in the regulation of cardiovascular function, body fluid balance, immune response, feeding, and reproduction; therefore, there are numerous possible outcomes for PK2 action. The early morning peak in PK2 expression that is coincident with the morning peak in cardiovascular activity (Guo and Stein, 2003) suggests that the SFO could have a regulatory role in conveying circadian rhythm signaling to other cardiovascular centers, and PK2 may be a factor in this. Future studies examining both the influence of PK2 on cardiovascular function and the SFO role in cardiovascular rhythms are warranted to clarify whether this association exists.

No previous electrophysiological experiments have assessed the effects of PK2 on neuronal excitability or ion channels. The inhibition of $I_{\mathrm{K}}$ current by PK2 reported here is novel, yet not unexpected. Washburn et al. (1999) demonstrated that vasopressin increased SFO neuron excitability in $65 \%$ of the neurons examined and inhibited both $I_{\mathrm{K}}$ and $I_{\mathrm{A}}$ currents. Similarly, Ferguson and $\mathrm{Li}(1996)$ demonstrated that angiotensin II inhibits $I_{\mathrm{A}}$ currents in SFO brain slices. In the present study, no effect of PK2 on $I_{\mathrm{A}}$ was found; however, the significant inhibition of $I_{\mathrm{K}}$ current likely contributed to enhanced neuronal excitability by increasing membrane potential at relatively depolarized states, prolonging action potentials, and decreasing the amplitude and duration of the afterhyperpolarization. Although some of these latter effects were observed during our current-clamp recordings, we chose to use more definitive voltage-clamp techniques to directly assess PK2 effects on $K_{\mathrm{v}}$ channels and, using such an approach, were able to identify clear specific effects on $I_{\mathrm{K}}$.

Voltage-clamp recordings in SFO neurons have previously characterized two populations of neurons, those that show a large degree of inactivation $\left(I_{\mathrm{A}}\right)$ and those that display a small degree of inactivation $\left(I_{\mathrm{K}}\right)$ (Anderson et al., 2001; Desson and Ferguson, 2003). The physiological relevance of this categorization is not clear; however, those SFO neurons projecting to the paraventricular nucleus are of the $I_{\mathrm{A}}$ type (Anderson et al., 2001). In the present experiments, PK2 effects were observed in both populations to an equal extent (data not shown); therefore, the PK2sensitive SFO neurons could not be categorized as specifically projecting to the PVN. Additional work must try to identify the characteristics of the PK2-responsive SFO neurons to better determine the excitatory role that PK2 has on these cells. The observation that only $40 \%$ of the neurons tested were PK2 responsive suggests that there is a distinct population of SFO neurons that may play a specific signaling role. Similar observations have been made for ANG (65\% responding), vasopressin (60\% responding), and IL1- $\beta$ ( $65 \%$ responding) actions in the SFO (Ferguson et al., 1997; Washburn et al., 1999; Desson and Ferguson, 2003), thus suggesting a heterogeneous neuronal population.

PK2 appears to fit into a class of peptides that were originally found associated with the gastrointestinal system but also have potent signaling effects in autonomic centers of the brain. These peptides include, but are not limited to, secretin, cholecystokinin, gastrin-releasing peptide, neuropeptide $Y$, vasointestinal peptide, bombesin, and ghrelin. The evolutionary need for the development of this diverse group of signaling peptides with multifactorial roles is not clear. However, it implies a close relationship between central and peripheral control of autonomic activity. 
In conclusion, we have demonstrated that PK2 behaves as an excitatory neuropeptide, depolarizes neurons of the SFO, and inhibits delayed rectifier potassium currents. These observations are the first to demonstrate that PK2 has a neuromodulatory role in the CNS and identifies the SFO as a potential site for circadian regulation.

\section{References}

Anderson JW, Smith PM, Ferguson AV (2001) Subfornical organ neurons projecting to paraventricular nucleus: whole-cell properties. Brain Res 921:78-85.

Bullock CM, Li JD, Zhou QY (2004) Structural determinants required for the bioactivities of prokineticins and identification of prokineticin receptor antagonists. Mol Pharmacol 65:582-588.

Cheng MY, Bullock CM, Li C, Lee AG, Bermak JC, Belluzzi J, Weaver DR, Leslie FM, Zhou QY (2002) Prokineticin 2 transmits the behavioural circadian rhythm of the suprachiasmatic nucleus. Nature 417:405-410.

Cottrell GT, Ferguson AV (2004) Sensory circumventricular organs: central roles in integrated autonomic regulation. Regul Pept 117:11-23.

Desson SE, Ferguson AV (2003) Interleukin 1 beta\} modulates rat subfornical organ neurons as a result of activation of a non-selective cationic conductance. J Physiol (Lond) 550:113-122.

Ferguson AV, Li Z (1996) Whole-cell patch clamp recordings from forebrain slices demonstrate angiotensin II inhibits potassium currents in subfornical organ neurons. Regul Pept 66:55-58.

Ferguson AV, Renaud LP (1986) Systemic angiotensin acts at subfornical organ to facilitate activity of neurohypophysial neurons. Am J Physiol 251:R712-R717.

Ferguson AV, Bicknell RJ, Carew MA, Mason WT (1997) Dissociated adult rat subfornical organ neurons maintain membrane properties and angiotensin responsiveness for up to 6 days. Neuroendocrinology 66:409-415.
Guo YF, Stein PK (2003) Circadian rhythm in the cardiovascular system: chronocardiology. Am Heart J 145:779-786.

LeCouter J, Kowalski J, Foster J, Hass P, Zhang Z, Dillard-Telm L, Frantz G, Rangell L, DeGuzman L, Keller GA, Peale F, Gurney A, Hillan KJ, Ferrara N (2001) Identification of an angiogenic mitogen selective for endocrine gland endothelium. Nature 412:877-884.

Li M, Bullock CM, Knauer DJ, Ehlert FJ, Zhou QY (2001) Identification of two prokineticin cDNAs: recombinant proteins potently contract gastrointestinal smooth muscle. Mol Pharmacol 59:692-698.

Lind RW, Van Hoesen GW, Johnson AK (1982) An HRP study of the connections of the subfornical organ of the rat. J Comp Neurol 210:265-277.

Masuda Y, Takatsu Y, Terao Y, Kumano S, Ishibashi Y, Suenaga M, Abe M, Fukusumi S, Watanabe T, Shintani Y, Yamada T, Hinuma S, Inatomi N, Ohtaki T, Onda H, Fujino M (2002) Isolation and identification of EGVEGF/prokineticins as cognate ligands for two orphan G-proteincoupled receptors. Biochem Biophys Res Commun 293:396-402.

McKinley MJ, Allen AM, Burns P, Colvill LM, Oldfield BJ (1998) Interaction of circulating hormones with the brain: the roles of the subfornical organ and the organum vasculosum of the lamina terminalis. Clin Exp Pharmacol Physiol Suppl 25:S61-S67.

Melchiorri D, Bruno V, Besong G, Ngomba RT, Cuomo L, De Blasi A, Copani A, Moschella C, Storto M, Nicoletti F, Lepperdinger G, Passarelli F (2001) The mammalian homologue of the novel peptide Bv8 is expressed in the central nervous system and supports neuronal survival by activating the MAP kinase/PI-3-kinase pathways. Eur J Neurosci 13:1694-1702.

Negri L, Lattanzi R, Giannini E, Metere A, Colucci M, Barra D, Kreil G, Melchiorri P (2002) Nociceptive sensitization by the secretory protein Bv8. Br J Pharmacol 137:1147-1154.

Washburn DLS, Beedle AM, Ferguson AV (1999) Inhibition of subfornical organ neuronal potassium channels by vasopressin. Neuroscience 93:349-359. 